\title{
Holographic three-point functions of semiclassical states
}

\section{K. Zarembo}

CNRS - Laboratoire de Physique Théorique, École Normale Supérieure, 24 rue Lhomond, 75231 Paris, France

Department of Physics and Astronomy, Uppsala University, SE-751 08 Uppsala, Sweden

E-mail: Konstantin.Zarembo@lpt.ens.fr

ABStract: We calculate the holographic three-point functions in $\mathcal{N}=4$ super-YangMills theory in the case when two of the operators are semiclassical and one is dual to a supergravity mode. We further discuss the transition to the regime when all three operators are semiclassical.

KEYwords: AdS-CFT Correspondence, Long strings

ARXiv EPRINT: 1008.1059

${ }^{1}$ Also at ITEP, Moscow, Russia 


\section{Contents}

1 Introduction 1

2 Holographic OPE 3

2.1 General formalism 3

$\begin{array}{lll}2.2 & \text { BMN operators } & 7\end{array}$

2.3 Spinning strings in $S^{5} \quad 7$

2.4 Saddle point approximation 9

$\begin{array}{lll}3 & \text { Fine structure of the spike } & 10\end{array}$

4 Discussion 13

$\begin{array}{ll}\text { A } \text { Spike in } S^{5} & 14\end{array}$

\section{Introduction}

The correlation functions in the $\mathcal{N}=4$ super-Yang-Mills theory (SYM) can be calculated both at weak and at strong coupling, owing to the AdS/CFT duality [1-3]. The two-point functions, by conformal symmetry, are determined by the spectrum of anomalous dimensions, which can be computed non-perturbatively with the help of integrability (see [4-6] for recent reviews), in the leading planar order of the large- $N$ expansion. The threepoint functions are the simplest observables of the next order in $1 / N$. The three-point functions of protected operators can be calculated at strong coupling in the supergravity approximation [7-11]. Non-protected operators with large quantum numbers constitute another potentially solvable case. At strong coupling they are dual to classical spinning strings [12-15], and there has been a renewed interest in computing their correlation functions holographically [16-18], but going beyond two-point correlators seems to be a difficult task. Here we consider an intermediate case when two operators in the correlator are semiclassical and one is protected. ${ }^{1}$ The three-point function can then be calculated with the help of the method developed in [20]. We will also discuss transition to the regime when all three states are semiclassical.

The three-point functions are determined by conformal symmetry up to an overall numerical factor. If the operators are scalar and conformal primary, their three-point functions have the form:

$$
\left\langle\mathcal{O}_{1}\left(x_{1}\right) \mathcal{O}_{2}\left(x_{2}\right) \mathcal{O}_{3}\left(x_{3}\right)\right\rangle=\frac{C_{123}}{\left|x_{1}-x_{2}\right|^{\Delta_{1}+\Delta_{2}-\Delta_{3}}\left|x_{1}-x_{3}\right|^{\Delta_{1}+\Delta_{3}-\Delta_{2}}\left|x_{2}-x_{3}\right|^{\Delta_{2}+\Delta_{3}-\Delta_{1}}} .
$$

\footnotetext{
${ }^{1}$ Similar calculations are done, using a different method, in a parallel independent publication [19].
} 


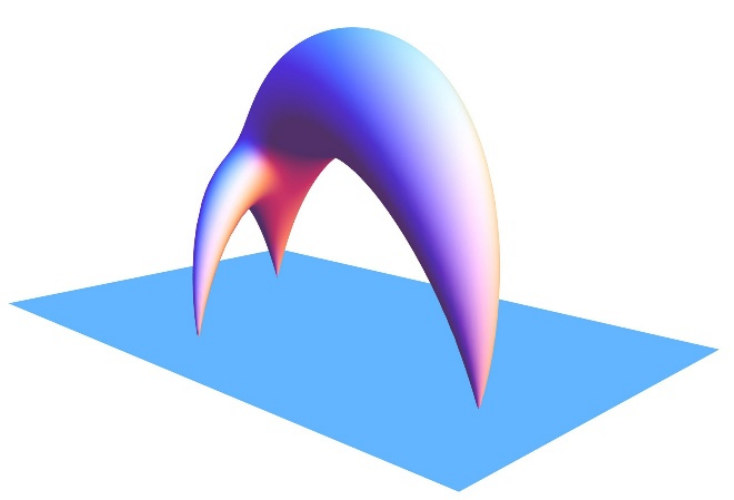

(a)

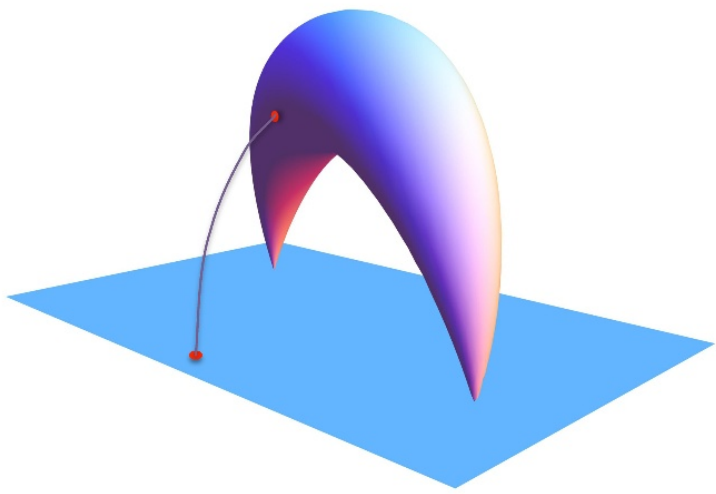

(b)

Figure 1. (a) The three-point function of semiclassical operators. (b) The correlator of two semiclassical operators and a supergravity mode.

The normalization here is important, and we will always assume that the two-point functions of conformal primaries are unit normalized:

$$
\left\langle\mathcal{O}_{I}^{\dagger}(x) \mathcal{O}_{J}(y)\right\rangle=\frac{\delta_{J}^{I}}{|x-y|^{2 \Delta_{I}}}
$$

The constant $C_{J K}^{I}$ then determines the coefficient with which the operator $\mathcal{O}_{I}$ appears in the operator product of $\mathcal{O}_{J}$ and $\mathcal{O}_{K}$ :

$$
\mathcal{O}_{J}(x) \mathcal{O}_{K}(0)=\sum_{I} C_{J K}^{I}|x|^{\Delta_{I}-\Delta_{J}-\Delta_{K}} \mathcal{O}_{I}(0)+\text { descendants. }
$$

On the string side of the duality, a SYM correlator is calculated by inserting vertex operators in the string path integral. The string tension $\sqrt{\lambda} / 2 \pi$ is large when the SYM 't Hooft coupling $\lambda$ is large, and at strong coupling the path integral is dominated by a saddle point. The semiclassical states are characterized by parametrically large scaling dimensions, $\Delta \propto \sqrt{\lambda}$, such that their vertex operators should be taken into account as sources in the equations of motion for the embedding coordinates in $A d S_{5} \times S^{5}$ [21]. The vertex operator insertions make the string shrink and approach the boundary of $A d S_{5}$ at $x_{1}, x_{2}, x_{3}$, as shown in figure $1 \mathrm{a}^{2}{ }^{2}$ The correlator is determined by the classical string action, and is thus exponential in $\sqrt{\lambda}$. The $x$-dependent factors in (1.1) are consistent with this exponential dependence on (since $\Delta_{I} \sim \sqrt{\lambda}$ ) and, as shown in [17], the correct space-time structure of the two- and three-point correlation functions indeed follows from the classical string calculation. The OPE coefficients of the semiclassical states should also be exponentially small (or large) at strong coupling: $-\ln C_{J K}^{I} \propto \sqrt{\lambda}$.

\footnotetext{
${ }^{2}$ The semiclassical picture of the holographic correlation functions has been discussed at length in the context of the $A d S_{3} / C F T_{2}$ correspondence [22].
} 
The classical string solutions that are dual to the two-point functions are relatively simple. They can be obtained by the Euclidean continuation of spinning strings in global AdS. In the Poincaré patch the string worldsheet indeed collapses onto the boundary at two points $[16,17,23]$. Constructing solutions dual to three-point functions seems to be a difficult problem. ${ }^{3}$ Here we study an intermediate case, when two strings are "fat" (dual to operators with $\Delta \propto \sqrt{\lambda}$ ) and one string is "slim" and particle-like (dual to an operator with $\Delta=O(1)$ ). The corresponding Witten diagram is shown in figure $1 \mathrm{~b}$. We further assume that the "fat" string is not much disturbed by the insertion of the "slim" vertex operator, which means that two of the operators in the correlator must be virtually the same. ${ }^{4}$ Then we only need to know the fat-string solution for the two-point function and the vertex operator of the slim string.

\section{Holographic OPE}

One of the operators in the correlation functions that we are going to compute will always be a chiral primary. The chiral primary operators in $\mathcal{N}=4$ SYM theory are symmetrized single-trace products of the six scalar fields from the $\mathcal{N}=4$ multiplet:

$$
\mathcal{O}_{I}^{\mathrm{CPO}}=\frac{1}{\sqrt{k}}\left(\frac{8 \pi^{2}}{\lambda}\right)^{\frac{k}{2}} K_{I}^{i_{1} \ldots i_{k}} \operatorname{tr} \Phi_{i_{1}} \ldots \Phi_{i_{k}} .
$$

The coefficients $K_{I}^{i_{1} \ldots i_{k}}$ are symmetric traceless tensors of $S O(6)$, which at the same time define the spherical functions on $S^{5}$ :

$$
Y_{I}(\mathbf{n})=K_{I}^{i_{1} \ldots i_{k}} n_{i_{1}} \ldots n_{i_{k}} .
$$

We assume the following normalization condition:

$$
K_{I}^{i_{1} \ldots i_{k}} K_{J}^{i_{1} \ldots i_{k}}=\delta_{I J}
$$

It guarantees the correct normalization of the two-point functions. Requiring orthonormality of the spherical functions leads to a different normalization condition, which is necessary to keep in mind in the holographic calculations. The rescaling factor is computed in [9].

\subsection{General formalism}

To compute the three-point functions of the chiral primary operators with semiclassical states, we will use the method applied in [20] to correlation functions of a local operator with a Wilson loop. The method can be generalized to any non-local operator $\mathcal{W}$ which is

\footnotetext{
${ }^{3}$ The classical decay process of certain folded string solution has been studied in the Minkowski signature [24]. The relationship of these Minkowski-signature solutions in global AdS to the three-point functions in SYM is not clear to us. On the one hand, as argued in [25], the holographic correlation functions are described by tunneling. The Euclidean signature in this respect is more natural [18]. On the other hand, the spinning string solutions with non-zero angular momenta in AdS approach the boundary at a point only if the worldsheet is Minkowskian [17], otherwise (in the Euclidean signature) the boundary maps to a line $[18,26]$.

${ }^{4}$ More precisely, they should be conjugate.
} 
dual to a classical string worldsheet. This can be a Wilson loop as in [20], or a product of local operators as in our case. Let us define the following ratio of correlation functions:

$$
\left\langle\mathcal{O}_{I}(x)\right\rangle_{\mathcal{W}}=\frac{\left\langle\mathcal{W} \mathcal{O}_{I}(x)\right\rangle}{\langle\mathcal{W}\rangle}
$$

where $\mathcal{O}_{I}$ is the local operator of interest, which is dual to a supergravity mode.

At large distances the correlator falls off as $|x|^{-2 \Delta_{I}}$, and we can extract the OPE coefficient

$$
\mathbb{C}_{I}[\mathcal{W}]=\lim _{x \rightarrow \infty}|x|^{2 \Delta_{I}}\left\langle\mathcal{O}_{I}(x)\right\rangle_{\mathcal{W}}
$$

Assuming that the operator $\mathcal{W}$ has a finite space-time support, the OPE coefficient $\mathbb{C}_{I}[\mathcal{W}]$ determines the amplitude to find $\mathcal{O}_{I}$ in the operator expansion of $\mathcal{W}$ around the origin:

$$
\mathcal{W}=\sum_{I} \mathbb{C}_{I}[\mathcal{W}] \mathcal{O}_{I}(0)+\text { descendants }
$$

When $\mathcal{W}$ is a product of two local operators, $\mathbb{C}_{I}$ is related to the conventional OPE coefficient from (1.1), (1.3):

$$
\mathbb{C}_{I}\left[\mathcal{O}_{J}^{\dagger}\left(x_{1}\right) \mathcal{O}_{K}\left(x_{2}\right)\right]=\left|x_{1}-x_{2}\right|^{\Delta_{I}} C_{I K}^{J} .
$$

How to calculate the correlation function (2.4) holographically? Keeping in mind that $\mathcal{O}_{I}$ is dual to a supergravity mode and $\mathcal{W}$ is dual to a classical string, the best suited formalism is a hybrid of the first-quantized string theory and supergravity:

$$
\left\langle\mathcal{O}_{I}(y)\right\rangle_{\mathcal{W}}=\lim _{\varepsilon \rightarrow 0} \frac{\pi}{\varepsilon^{\Delta_{I}}} \sqrt{\frac{2}{\Delta_{I}-1}}\left\langle\phi_{I}(y, \varepsilon) \frac{1}{Z_{\text {str }}} \int \mathcal{D} \mathbb{X} \mathrm{e}^{-S_{\text {str }}[\mathbb{X}]}\right\rangle_{\text {bulk }}
$$

The integration variables $\mathbb{X}$ in the string path integral are the embedding coordinates of the string worldsheet in $A d S_{5} \times S^{5}$ and, in principle, fermions, but in the semiclassical approximation the fermions will not be important. For the AdS metric we take

$$
d s^{2}=\frac{d x_{\mu}^{2}+d z^{2}}{z^{2}}
$$

and parameterize $S^{5}$ by a unit $6 \mathrm{~d}$ vector $\mathbf{n}$. The boundary conditions on the string worldsheet are determined by the non-local operator $\mathcal{W}$. The supergravity field $\phi_{I}(x, z)$ is dual to the local operator $\mathcal{O}_{I}$, and the bulk average is defined by the action (interactions in the bulk are $1 / N$ suppressed):

$$
S_{\mathrm{bulk}}=\frac{1}{2} \int d^{4} x \frac{d z}{z^{5}}\left[\left(\partial \phi_{I}\right)^{2}+\Delta_{I}\left(\Delta_{I}-4\right) \phi_{I}^{2}\right] .
$$

The field $\phi_{I}$ has the standard AdS propagator

$$
\left\langle\phi_{I}(x, z) \phi_{I}(y, w)\right\rangle_{\mathrm{bulk}}=\frac{\Delta_{I}-1}{2 \pi^{2}} \frac{z^{\Delta_{I}} w^{\Delta_{I}}}{\left[z^{2}+(x-y)^{2}\right]^{\Delta_{I}}}\left(1+O\left(w^{2}\right)\right) .
$$


The string action,

$$
S_{\mathrm{str}}=\frac{\sqrt{\lambda}}{4 \pi} \int d^{2} \sigma \sqrt{h} h^{\mathbf{a b}} \partial_{\mathbf{a}} \mathbb{X}^{M} \partial_{\mathbf{b}} \mathbb{X}^{N} G_{M N}+\ldots
$$

depends on the supergravity modes $\phi_{I}$ indirectly, through the disturbance of the metric created by the local operator insertion, which we denote by $\gamma_{M N}$ :

$$
G_{M N}=g_{M N}+\gamma_{M N}
$$

where $g_{M N}$ is the unperturbed metric of $A d S_{5} \times S^{5}$. For brevity we have omitted the coupling to $B_{M N}$, the dilaton, and the fermions. The response of the metric to a perturbation in $\phi_{I}$ depends on the details of the Kaluza-Klein reduction of the 10d supergravity on $S^{5}$ [27]. In general, the 10d metric perturbation is a linear combination of the normal modes $\phi_{I}$ and their derivatives:

$$
\gamma_{M N}=V_{M N}^{I} \phi_{I}
$$

where $V_{M N}^{I}$ is a second-order differential operator with $\mathbb{X}$-dependent coefficients.

At large $\lambda$ the string path integral is dominated by a saddle point, and we can substitute the classical solution for $\mathbb{X}^{M}=\left(z(\sigma), x^{\mu}(\sigma), \mathbf{n}(\sigma)\right)$, expand the string action to the linear order in $\phi_{I}$, and use the propagator (2.11) to calculate the bulk expectation value:

$$
\begin{aligned}
\left\langle\mathcal{O}_{I}(y)\right\rangle_{\mathcal{W}}= & -\frac{\sqrt{2\left(\Delta_{I}-1\right) \lambda}}{8 \pi^{2}} \int d^{2} \sigma \sqrt{h} h^{\mathbf{a b}} \partial_{\mathbf{a}} \mathbb{X}^{M} \partial_{\mathbf{b}} \mathbb{X}^{N} \\
& \times V_{M N}^{I}\left(\mathbb{X}, \frac{\partial}{\partial x}, \frac{\partial}{\partial z}\right) \frac{z^{\Delta_{I}}}{\left[z^{2}+(x-y)^{2}\right]^{\Delta_{I}}}
\end{aligned}
$$

The answer has the form of a vertex operator in the coordinate representation $[28,29]$ integrated over the classical string worldsheet.

We can now take $y$ to infinity, to determine the OPE coefficient $\mathbb{C}_{I}[\mathcal{W}]$. The vertex operator then simplifies a bit. In particular, $\partial / \partial x$ can be set to zero [20], because differentiating in $x$ increases the power of $y$ and thus only contributes to correlators with the descendants of $\mathcal{O}_{I}(y)$ :

$$
\mathbb{C}_{I}[\mathcal{W}]=-\frac{\sqrt{2\left(\Delta_{I}-1\right) \lambda}}{8 \pi^{2}} \int d^{2} \sigma \sqrt{h} h^{\mathbf{a b}} \partial_{\mathbf{a}} \mathbb{X}^{M} \partial_{\mathbf{b}} \mathbb{X}^{N} V_{M N}^{I}\left(\mathbb{X}, \frac{\partial}{\partial z}\right) z^{\Delta_{I}}
$$

Throughout this paper we will use the conformal gauge for the classical solutions, and later the Weyl-invariant factor $\sqrt{h} h^{\mathbf{a b}}$ will be replaced by $\delta^{\mathbf{a b}}$.

In the case when $\mathcal{O}_{I}$ is the chiral primary operator $\mathcal{O}_{I}^{\mathrm{CPO}}$, the dual supergravity field $s_{I}$ is a mixture of the metric with the RR four-form. The metric perturbation (2.14) can be written explicitly after decomposing the 10d index into the tangent-space indices of $A d S_{5}$ $(m, n, \ldots)$ and $S^{5}(\alpha, \beta, \ldots): M=(m, \alpha)$. The Kaluza-Klein reduction on $S^{5}$ [27] gives [9] 
(see also [30]):

$$
\begin{aligned}
h_{m n} & =\frac{1}{\mathcal{N}_{k}} \frac{2}{k+1} Y_{I}\left[2 \nabla_{m} \nabla_{n}-k(k-1) g_{m n}\right] s_{I} \\
h_{\alpha \beta} & =\frac{2}{\mathcal{N}_{k}} k g_{\alpha \beta} Y_{I} s_{I} \\
a_{\alpha \beta \gamma \delta} & =-\frac{1}{\mathcal{N}_{k}} \varepsilon_{\alpha \beta \gamma \delta \varepsilon} \nabla^{\varepsilon} Y_{I} s_{I} \\
a_{m n p r} & =\frac{1}{\mathcal{N}_{k}} \varepsilon_{m n p r s} Y_{I} \nabla^{s} s_{I},
\end{aligned}
$$

where $Y_{I} \equiv Y_{I}(\mathbf{n})$ are the spherical functions (2.2). The common normalization factor,

$$
\mathcal{N}_{k}^{2}=\frac{N^{2} k(k-1)}{2^{k-3} \pi^{2}(k+1)^{2}}
$$

takes into account the $10 \mathrm{~d}$ gravitational constant, which appears in front of the supergravity action and is equal to $\kappa_{10}^{2}=(2 \pi)^{5} / 8 N^{2}$ in the units of the AdS radius, the mixing effects in the KK reduction, and the unusual normalization of the spherical functions. The OPE coefficients owe their universal $1 / N$ dependence on $N$ precisely to this normalization factor (and ultimately to the $10 \mathrm{~d}$ gravitational constant).

The RR field couples only to fermions which at the classical level can be neglected. Substituting the metric from (2.17) into the string action we finally get for the OPE coefficient $^{5}$

$$
\mathbb{C}_{I}^{\mathrm{CPO}}[\mathcal{W}]=\frac{2^{\frac{k}{2}-3}(k+1) \sqrt{k \lambda}}{\pi N} \int d^{2} \sigma Y_{I}(\mathbf{n})\left[z^{k-2}(\partial x)^{2}-z^{k-2}(\partial z)^{2}-z^{k}(\partial \mathbf{n})^{2}\right] .
$$

We are going to use this formula to compute the three-point functions of various semiclassical operators with the BMN-type chiral primary,

$$
\mathcal{O}_{k}=\frac{1}{\sqrt{k}}\left(\frac{4 \pi^{2}}{\lambda}\right)^{\frac{k}{2}} \operatorname{tr} Z^{k}, \quad Z=\Phi_{1}+i \Phi_{2} .
$$

This operator is the highest-weight state in the $[0, k, 0]$ representation of $S O(6)$. In the standard angular parameterization of the five-sphere,

$$
\begin{aligned}
\mathbf{n}= & (\sin \theta \cos \varphi, \sin \theta \sin \varphi, \cos \theta \sin \alpha \cos \psi, \cos \theta \sin \alpha \sin \psi, \\
& \cos \theta \cos \alpha \cos \beta, \cos \theta \cos \alpha \sin \beta),
\end{aligned}
$$

the associated spherical function is

$$
Y_{k}=\left(\frac{n_{1}+i n_{2}}{\sqrt{2}}\right)^{k}=2^{-\frac{k}{2}}(\sin \theta)^{k} \mathrm{e}^{i k \varphi}
$$

\footnotetext{
${ }^{5}$ In passing from (2.16) to this equation the partial derivatives in $x$ can be dropped and the covariant derivatives $\nabla_{\mu}$ are replaced by the Christoffel symbols.
} 


\section{$2.2 \quad$ BMN operators}

As a check on the formalism we first consider the case when the other two operators are also chiral primaries, but carry large quantum numbers. The OPE coefficients of three chiral primary operators are actually known exactly [9], since they are not renormalized and do not depend on $\lambda[9,31-35]$ (but there are non-trivial $1 / N$ corrections [36]). For the BMN primaries (2.20) the OPE coefficients are equal to

$$
C_{k_{2} k_{3}}^{k_{1}}=\frac{1}{N} \sqrt{k_{1} k_{2} k_{3}}
$$

where the condition $k_{1}=k_{2}+k_{3}$ must be imposed to satisfy the R-charge conservation.

We can compute $C_{J k}^{J+k}$ at strong coupling in the regime when two operators are fat and one is slim:

$$
J=O(\sqrt{\lambda}), \quad k=O(1) .
$$

The two-point function $\left\langle\mathcal{O}_{J}^{\dagger} \mathcal{O}_{J}\right\rangle$ then is describes by the classical string solution [17, 23]:

$$
\begin{aligned}
x & =R \tanh \kappa \tau, \quad R=\frac{\left|x_{1}-x_{2}\right|}{2} \\
z & =\frac{R}{\cosh \kappa \tau} \\
\varphi & =i \kappa \tau, \quad \theta=\frac{\pi}{2} .
\end{aligned}
$$

which is basically the Euclidean continuation of the BMN geodesic [37]. The parameter $\kappa$ is related to the $\mathrm{R}$-charge/dimension of the fat operators:

$$
\kappa=\frac{J}{\sqrt{\lambda}} .
$$

Substituting this solution into (2.19) we find that the OPE coefficient scales with the distance in the right way: $\mathbb{C}_{k}\left[\mathcal{O}_{J+k}^{\dagger}\left(x_{1}\right) \mathcal{O}_{J}\left(x_{2}\right)\right] \propto R^{k}=2^{-k}\left|x_{1}-x_{2}\right|^{k}$, which agrees with the conformal structure of the three-point function. Pulling out the factor of $\left|x_{1}-x_{2}\right|^{k}$ according to (2.7), and performing integration over the classical worldsheet we find:

$$
C_{J, k}^{J+k}=\frac{1}{N} 2^{-k-1} J(k+1) \sqrt{k} \kappa \int_{-\infty}^{+\infty} d \tau \frac{\mathrm{e}^{-k \kappa \tau}}{\cosh ^{k+2} \kappa \tau}=\frac{1}{N} J \sqrt{k},
$$

in agreement with the exact result - in the regime (2.24) we cannot distinguish $J$ and $J+k$.

\subsection{Spinning strings in $S^{5}$}

When the fat operators are dual to a spinning string on $S^{5}$, the AdS part of the solution for the two-point function is again described by the point-like geodesic (2.25). In $S^{5}$ the worldsheet is a periodic solution of the $O(6)$ sigma-model $\mathbf{n}(\sigma, \tau)$. The OPE coefficient with the BMN chiral primary is given by (2.19), with (2.22) substituted for the spherical function:

$$
\mathbb{C}_{k}=\frac{(k+1) \sqrt{k \lambda}}{8 \pi N} \int d^{2} \sigma z^{k} \mathrm{e}^{i k \varphi} \sin ^{k} \theta\left[\frac{(\partial x)^{2}-(\partial z)^{2}}{z^{2}}-(\partial \mathbf{n})^{2}\right]
$$


Using the explicit form of the AdS geodesic and excluding $(\partial \mathbf{n} / \partial \sigma)^{2}$ with the help of the Virasoro constraints, we find, after extracting the factor of $\left|x_{1}-x_{2}\right|^{k}$ :

$$
C_{k}=-\frac{(k+1) \sqrt{k \lambda}}{2^{k+2} \pi N} \int_{-\infty}^{+\infty} \frac{d \tau}{\cosh ^{k} \kappa \tau} \int_{0}^{2 \pi} d \sigma \mathrm{e}^{i k \varphi} \sin ^{k} \theta\left[\kappa^{2} \tanh ^{2} \kappa \tau+\left(\frac{\partial \mathbf{n}}{\partial \tau}\right)^{2}\right] .
$$

The periodic solutions of the $O(6)$ sigma-model can be constructed in full generality with the help of integrability methods [38, 39]. The dual operators can then be identified through the Bethe ansatz equations. In principle the general finite-gap solution is know in a relatively explicit form [40], but not explicit enough to calculate the integral (2.30). Here we will compute the OPE coefficient for the folded string solution. The starting point is the string that rotates on the big circle of $S^{5}$ and spins around its centre of mass [41], which then should be Wick rotated to the Euclidean signature. The dual operator corresponds to the two-cut solution of the classical Bethe equations [42].

The folded string solution is characterized by two frequencies $\omega_{1}, \omega_{2}$ of rotation around $S^{5}$ and around the string's centre of mass:

$$
\varphi=i \omega_{1} \tau, \quad \psi=i \omega_{2} \tau, \quad \alpha=\frac{\pi}{2}, \quad \theta=\theta(\sigma), \quad \dot{\theta}^{2}=\kappa^{2}-\omega_{1}^{2} \sin ^{2} \theta-\omega_{2}^{2} \cos ^{2} \theta .
$$

The $\sigma$ dependence can be integrated in terms of elliptic functions whose modulus $s$ is related to the frequencies by

$$
s=\frac{\kappa^{2}-\omega_{1}^{2}}{\omega_{2}^{2}-\omega_{1}^{2}} .
$$

The periodicity in $\sigma$ relates $\omega_{1}, \omega_{2}$ and $\kappa$ :

$$
\sqrt{\omega_{2}^{2}-\omega_{1}^{2}}=\frac{2 K(s)}{\pi}
$$

where $K(s)$ is the complete elliptic integral of the first kind.

Instead of the frequencies, it is more convenient to characterize the solution by the conserved quantum numbers, the two angular momenta and the energy:

$$
J_{1}=\frac{\sqrt{\lambda} \omega_{1} E(s)}{K(s)}, \quad J_{2}=\frac{\sqrt{\lambda} \omega_{2}(K(s)-E(s))}{K(s)}, \quad \Delta=\sqrt{\lambda} \kappa,
$$

where $E(s)$ is the complete elliptic integral of the second kind. The angular momenta $J_{1}$ and $J_{2}$ are the Noether charges associated with the isometries $\varphi \rightarrow \varphi+$ const, $\psi \rightarrow \psi+$ const . The dual operator has the form $\operatorname{tr} Z^{J_{1}} W^{J_{2}}+$ permutations, $Z=\Phi_{1}+i \Phi_{2}, W=\Phi_{3}+i \Phi_{4}$, and $\Delta$ is its exact scaling dimension. Eqs. (2.32) and (2.33) express the dimension as a function of the R-charges in an implicit form: $\Delta=\Delta\left(J_{1}, J_{2}\right)$. Finally, it is useful to introduce a parameter

$$
a=\frac{\omega_{1}}{\kappa}=\frac{J_{1} K(s)}{\Delta E(s)} .
$$

The the integral in (2.30) can be calculated in terms of the hypergeometric function:

$$
\begin{aligned}
C_{\text {fold. }, k}^{\text {fold. }}= & \frac{1}{N} \frac{\pi \sqrt{k} \Delta\left(1-a^{2}\right) \Gamma\left(\frac{(1+a) k}{2}\right) \Gamma\left(\frac{(1-a) k}{2}\right)}{8 s K(s)(k-1) !} \\
& \times\left[(k+1-s)_{2} F_{1}\left(-\frac{k-1}{2}, \frac{1}{2} ; 1 ; s\right)-(k+1)_{2} F_{1}\left(-\frac{k+1}{2}, \frac{1}{2} ; 1 ; s\right)\right](2.3
\end{aligned}
$$


At $a \rightarrow 1$, the folded string shrinks to a point and goes over to the point-like solution dual to the chiral primary operator. However, we do not recover the OPE coefficient of three chiral primaries (2.28) in the limit $a \rightarrow 1$. There is an extra factor of $(k-1) / 2 k$. Why? The difference can be attributed to the anomaly that arises when the slim string vertex operator approaches the fat string vertex operator at $\tau=-\infty$. The integrand in (2.29) contains an exponential factor $\mathrm{e}^{i k \varphi} z^{k} \sim \mathrm{e}^{k\left(\kappa-\omega_{1}\right) \tau}$ that cuts off the integral at large negative $\tau$, because $\kappa>\omega_{1}$. In the point-string limit $\kappa \rightarrow \omega_{1}$ the suppression disappears. The square bracket decomposes in the limit in two terms. One is additionally suppressed as $\mathrm{e}^{2 \kappa \tau}$ and survives the BMN limit, making the $\tau$ integral in (2.28) manifestly convergent. The other term is proportional to $\dot{\theta}^{2} \sim \kappa-\omega_{1}$ and vanishes as the string becomes point-like, but it does not have the additional exponential suppression and integration in $\tau$ diverges in the limit leading to the $0 / 0$ cancelation.

\subsection{Saddle point approximation}

At large $k$ the integral over the vertex operator insertion in (2.29) is dominated by a saddle point, a solution of the equation

$$
\cot \theta \partial_{\mathbf{a}} \theta+i \partial_{\mathbf{a}} \varphi+\frac{\partial_{\mathbf{a}} Z}{Z}=0
$$

This constitutes a set of two equations on two variables and consequently is satisfied in a finite number of points. The OPE coefficient then is exponentially suppressed:

$$
C_{k} \sim \mathrm{e}^{-k S}
$$

where $S=-\ln \sin \theta\left(\sigma_{*}\right)-i \varphi\left(\sigma_{*}\right)-\ln Z\left(\sigma_{*}\right)$ and $\sigma_{*}=\left(\tau_{*}, \sigma_{*}\right)$ is the solution of (2.37) with the smallest possible suppression.

For instance, the folded string OPE with the chiral primary is exponentially small at large $k$ :

$$
C_{\text {fold. }, k}^{\text {fold. }} \simeq \frac{1}{N}\left(\frac{1}{s}-1\right) \sqrt{\frac{\lambda}{k}} \exp \left[k\left(\frac{1+a}{2} \ln \frac{1+a}{2}+\frac{1-a}{2} \ln \frac{1-a}{2}\right)\right]
$$

For the folded string there are two degenerate saddle points:

$$
\tau_{*}=\frac{1}{2 \kappa} \ln \frac{1-a}{1+a}, \quad \sigma_{*}=\frac{\pi}{2} \text { and } \frac{3 \pi}{2}: \theta\left(\sigma_{*}\right)=\frac{\pi}{2} .
$$

The pre-exponential factor vanished at the saddle point and has to be expanded to the second order to get the correct coefficient in front. It is actually easier to compute the integral exactly and then take the large- $k$ limit. In the point-like string limit of $a \rightarrow 1$, the saddle point (2.40) approaches the boundary and the exponential suppression disappears.

Our calculations were accurate at $k=O(1)$, but the limit of $k \rightarrow \infty$ should have an overlapping region of validity $\sqrt{\lambda} \gg k \gg 1$ with the $k / \sqrt{\lambda} \rightarrow 0$ limit of the OPE with all three string states being semiclassical. ${ }^{6}$ Because we took the limit $x_{3} \rightarrow \infty$ in the three-point function, the vertex operator of a fat string with $k \sim \sqrt{\lambda}$ creates an infinite spike on the worldsheet with two other vertex insertions ending on the boundary of AdS, 


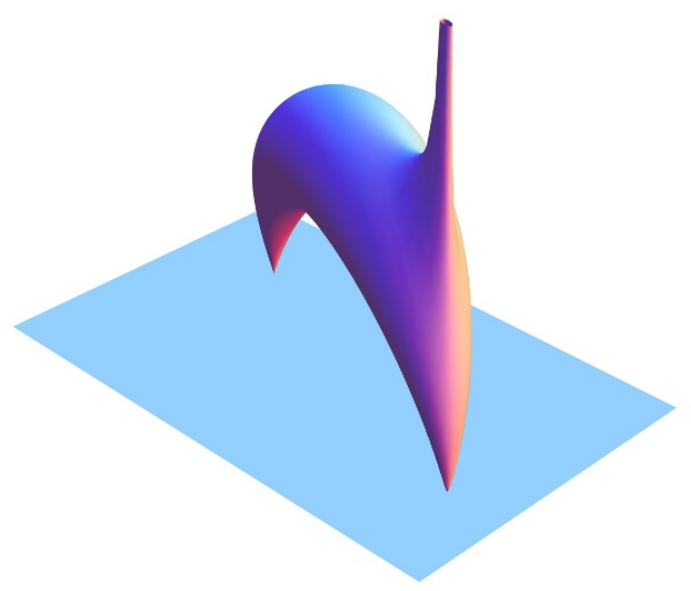

Figure 2. The vertex operator creates an infinite spike.

figure 2. When $k / \sqrt{\lambda} \rightarrow 0$ the spike shrinks to zero size. The saddle point $\sigma_{*}$ determines the position where the spike is attached to the macroscopic worldsheet, or better to say, the position where the equations of motion allow the spike to appear when we gradually raise $k / \sqrt{\lambda}$ from zero. We are going to demonstrate this explicitly in the next section, by starting with finite $k / \sqrt{\lambda}$ and then taking the limit of $k / \sqrt{\lambda} \rightarrow 0$.

\section{Fine structure of the spike}

Now we consider the regime when we insert the vertex operator (2.22) with $k \sim \sqrt{\lambda}$ in the string path integral. When $k \sim \sqrt{\lambda}$ the vertex operators changes the boundary conditions for the embedding coordinates and creates an infinite spike [43, 44] (figure 2). There may be other vertex operators or Wilson loops, which we do not specify, as we will be interested in the structure of the solution in the vicinity of the insertion point. The local worldsheet coordinates will be denoted by $w, \bar{w}$, and we will assume that the vertex operator is inserted at $w=0$.

At $\lambda \rightarrow \infty$, the logarithm of the vertex operator is of the same order as the action: ${ }^{7}$

$S=\frac{\sqrt{\lambda}}{4 \pi} \int d^{2} w\left[\frac{(\partial z)^{2}+(\partial x)^{2}}{z^{2}}+(\partial \theta)^{2}+\sin ^{2} \theta(\partial \varphi)^{2}\right]-k \ln z(0)-i k \varphi(0)-k \ln \sin \theta(0)$.

We do not display other coordinates or other vertex operators as we will be interested in the most singular behavior near $w=0$.

\footnotetext{
${ }^{6}$ This has been explicitly verified for the Wilson loop correlator with a local operator [43, 44].

${ }^{7}$ The action is written in the conformal gauge. The dependence on the $2 \mathrm{~d}$ metric should also drop from the vertex operator due to its marginality.
} 


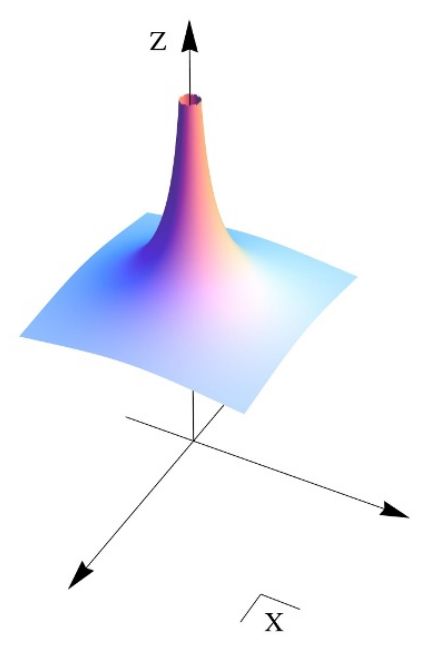

Figure 3. The string worldsheet in the vicinity of a spike.

The vertex operator creates a localized source in the equations of motion:

$$
\begin{aligned}
\partial_{\mathbf{a}}\left(\frac{\partial^{\mathbf{a}} x^{\mu}}{z^{2}}\right) & =0 \\
-\partial^{2} \ln z-\frac{(\partial x)^{2}}{z^{2}} & =2 \pi \chi \delta(w) \\
-\partial^{2} \theta+\sin \theta \cos \theta(\partial \varphi)^{2} & =2 \pi \chi \cot \theta \delta(w) \\
-\partial_{\mathbf{a}}\left(\sin ^{2} \theta \partial^{\mathbf{a}} \varphi\right) & =2 \pi i \chi \delta(w),
\end{aligned}
$$

where

$$
\chi=\frac{k}{\sqrt{\lambda}} .
$$

The source determines the boundary conditions for the worldsheet fields at $w=0$ :

$$
\begin{aligned}
\ln z & \rightarrow-\chi \ln |w| \\
\theta & \rightarrow \frac{\pi}{2} \\
\varphi & \rightarrow-i \chi \ln |w| .
\end{aligned}
$$

Let us see in more detail what is the structure of the classical worldsheet in the vicinity of $w=0$.

We begin with the shape of the string in AdS. According to the boundary conditions, the worldsheet has an infinite spike going all the way to the horizon (figure 3), with the AdS $z$ coordinate scaling as $z \sim|w|^{-\chi}$. As far as the $x^{\mu}$ coordinates are concerned, we can try an axially symmetric scaling ansatz $x \sim|w|^{\alpha} w$, where $x=x^{1}+i x^{2}$ is the complex coordinate on the boundary of $A d S_{3}$. The ansatz goes through the equations if $\alpha$ satisfies:

$$
\frac{\alpha}{2}\left(\frac{\alpha}{2}+1+\chi\right)+\left(\frac{\alpha}{2}+1\right)\left(\frac{\alpha}{2}+\chi\right)=0,
$$

giving

$$
\alpha=\sqrt{1+\chi^{2}}-1-\chi
$$


It is easy to understand the meaning of this result by making the conformal map to the cylinder:

$$
w=\mathrm{e}^{-\tau+i \sigma} .
$$

The transformation $z \rightarrow \mathrm{e}^{\chi \tau} z, x \rightarrow \mathrm{e}^{-\chi \tau} x$ generates a mass term of magnitude $\chi$ in the equations of motion for $x$. The frequency of the $n$th Fourier mode of a $2 \mathrm{~d}$ field of mass $\chi$ is equal to $\omega_{n}=\sqrt{n^{2}+\chi^{2}}$. The axially symmetric solution corresponds to the single-winding mode $x \sim \mathrm{e}^{-\omega_{1} \tau+i \sigma}$.

The rate at which $x$ goes to zero at $w \rightarrow 0$ is always larger than the rate at which $\ln z$ goes to infinity. For this reason $x$ does not backreact on $z$ up to the next-to-next-toleading order. It is therefore easy to deduce the next term in the expansion of $z$ in $w$ by just treating $\ln z$ as a free massless field with the source at the origin. The most general solution then reads:

$$
\begin{aligned}
z & =|w|^{-\chi}\left(z_{0}+\partial z_{0} w+\bar{\partial} z_{0} \bar{w}\right)+\ldots \\
x^{\mu} & =x_{0}^{\mu}+|w|^{\sqrt{1+\chi^{2}}-1-\chi}\left(\partial x_{0}^{\mu} w+\bar{\partial} x_{0}^{\mu} \bar{w}\right)+\ldots,
\end{aligned}
$$

where $z_{0}, x_{0}^{\mu}, \partial z_{0}, \bar{\partial} z_{0}, \partial x_{0}^{\mu}$, and $\bar{\partial} x_{0}^{\mu}$ are constants, not determined by the equations of motion.

Now we can take the $\chi \rightarrow 0$ limit. The spike then disappears, and (3.7) becomes an ordinary Taylor expansion of the regular solution without the source at $w=0$. We can thus regard $\left(z_{0}, x_{0}^{\mu}\right)$ as the target-space coordinates of the point at which the spike is attached to the string worldsheet. The constants $\partial z_{0}, \ldots$ are Taylor expansion coefficients of the smooth solution $\left(z_{0}(w), x_{0}^{\mu}(w)\right)$ for the string without the spike.

We now turn to the $S^{5}$. The boundary conditions imply that $\mathrm{e}^{i \varphi} \rightarrow$ const $|w|^{\chi}$ and $\cos \theta \rightarrow \operatorname{const}|w|^{\chi}$ at $w \rightarrow 0$. Corrections to this behavior are of two types. There are regular corrections in powers of $w, \bar{w}$, as well as non-analytic corrections in $|w|^{\chi}$. Which of those are more important depends on the value of $\chi$. When $\chi \rightarrow 0$ and the spike shrinks leaving behind some regular solution $\theta_{0}(w), \varphi_{0}(w)$, the non-analytic corrections are more important, moreover in the strict $\chi \rightarrow 0$ limit they cease to be suppressed and have to be re-summed. It is possible to do the re-summation explicitly, although the details are somewhat lengthy. The solution which is accurate to all orders in $|w|^{\chi}$ and to the first two orders in $w, \bar{w}$ is derived in the appendix $\mathrm{A}$ :

$$
\begin{aligned}
\mathrm{e}^{i \varphi}= & \frac{|w|^{\chi} \mathrm{e}^{i \varphi_{0}} \sin \theta_{0}}{\sqrt{1-|w|^{2 \chi} \cos ^{2} \theta_{0}}}\left[1+i \frac{1-|w|^{\chi} \cos ^{2} \theta_{0}}{1-|w|^{2 \chi} \cos ^{2} \theta_{0}}\left(\partial \varphi_{0} w+\bar{\partial} \varphi_{0} \bar{w}\right)\right. \\
& \left.+\frac{\left(1-|w|^{\chi}\right) \cot \theta_{0}}{1-|w|^{2 \chi} \cos ^{2} \theta_{0}}\left(\partial \theta_{0} w+\bar{\partial} \theta_{0} \bar{w}\right)\right]+\ldots \\
\cos \theta= & |w|^{\chi} \cos \theta_{0}-|w|^{\sqrt{1+\chi^{2}}-1}\left[\frac{1-|w|^{\chi} \cos ^{2} \theta_{0}}{\sin \theta_{0}}\left(\partial \theta_{0} w+\bar{\partial} \theta_{0} \bar{w}\right)\right. \\
& \left.+i\left(1-|w|^{\chi}\right) \cos \theta_{0}\left(\partial \varphi_{0} w+\bar{\partial} \varphi_{0} \bar{w}\right)\right]+\ldots,
\end{aligned}
$$

where again $\theta_{0}, \varphi_{0}, \partial \theta_{0}, \ldots$ are constants not fixed by the equations of motion. These constants can be regarded as the Taylor coefficients of the regular solution. The above 
approximate solution is valid in the limit of $w \rightarrow 0$ (in that case all subleading $|w|^{n \chi}$ terms can be dropped), as well as in the double-scaling limit $w \rightarrow 0, \chi \rightarrow 0$, with $|w|^{\chi}$ fixed (in which case $|w|^{\sqrt{1+\chi^{2}}-1}$ should be replaced by 1$)$.

To the leading order in $w$, the solution (3.7), (3.8) automatically satisfies the Virasoro constraints, which reflects the marginality of the vertex operator (2.22). At the next order the Virasoro constraints are not automatic and lead to the relationship between the expansion coefficients $\partial z_{0}, \ldots$

Requiring that

$$
0=T_{w w} \equiv\left(\frac{\partial z}{z}\right)^{2}+(\partial \theta)^{2}+\sin ^{2} \theta(\partial \varphi)^{2},
$$

and imposing the same condition on $T_{\bar{w} \bar{w}}$, we find a linear relationship between the coefficients:

$$
\frac{\partial z_{0}}{z_{0}}+\cot \theta_{0} \partial \theta_{0}+i \partial \varphi_{0}=0, \quad \frac{\bar{\partial} z_{0}}{z_{0}}+\cot \theta_{0} \bar{\partial} \theta_{0}+i \bar{\partial} \varphi_{0}=0
$$

Viewed as a condition on the unperturbed solution $\theta_{0}, \varphi_{0}$, it determines a point on the worldsheet to which the spike can be attached without violating the Virasoro constraints. This condition is the same as the saddle point equation (2.37), which was derived when we neglected the backreaction of the vertex operator on the shape of the string.

\section{Discussion}

It would be interesting to compare the three-point functions computed at weak and at strong coupling, in particular to check if the structural observations made recently in the one-loop corrections to the three-point functions of scalar operators [45] survive at strong coupling. It would also be interesting to generalize integrability methods to three-point and higher correlation functions. The spectral equations derived using integrability methods determine the eigenvalues of the light-cone string Hamiltonian. To compute correlation functions, which are analogous to closed string amplitudes in the familiar flat-space setting, one also needs to know the wavefunctions, which are more or less equivalent to vertex operators. The vertex operators of the chiral states can be deduced from the supergravity equations of motion, and probably do not receive $\alpha^{\prime}(1 / \sqrt{\lambda})$ corrections. It would be interesting to understand how to systematically construct vertex operators of non-protected states $[18,29]$. Then one will be able to compute their holographic three-point functions, at least in the approximation used in this paper.

\section{Acknowledgments}

I would like to thank R. Janik and V. Kazakov for collaboration on the initial stages of this project and for numerous discussions. I benefited a lot from the discussions with J. Escobedo, I. Kostov, D. Serban, A. Sever and P. Vieira. I would like to thank M. Costa, R. Monteiro, J. Santos and D. Zoakos for sending me the draft of [19] prior to publication, and the Perimeter Institute for hospitality during the course of this project. This work was supported in part by the Swedish Research Council under grant 621-2007-4177, in part 
by the ANF-a grant 09-02-91005, and in part by the grant for support of scientific schools NSH-3036.2008.2.

\section{A Spike in $S^{5}$}

It is easier to analyze the solution in the polar coordinates (3.6). To the leading order, the solution depends only on $\tau$, and the equations of motion become ordinary differential equations:

$$
\begin{aligned}
\left(\sin ^{2} \theta \dot{\varphi}\right) & =0 \\
\ddot{\theta}-\sin \theta \cos \theta \dot{\varphi}^{2} & =0 .
\end{aligned}
$$

The boundary conditions (3.4) require that $\varphi \rightarrow i \chi \tau, \theta \rightarrow \pi / 2$ at $\tau \rightarrow \infty$. Taking this into account we find the solution that depends on the two integration constants:

$$
\begin{aligned}
\cos \theta & =\mathrm{e}^{-\chi \tau} \cos \theta_{0} \\
\mathrm{e}^{i \varphi} & =\mathrm{e}^{i \varphi_{0}} \cot \theta .
\end{aligned}
$$

The next order in $w, \bar{w}$ corresponds to the first Kaluza-Klein mode in $\sigma$ and is suppressed by an extra factor of $\mathrm{e}^{-\tau}$, which is a small correction at $\tau \rightarrow \infty$. The equations of motion can be consequently linearized.

We consider a slightly more general setup when the linear correction is the $n$th harmonic. Substituting

$$
\delta \theta=\xi \mathrm{e}^{-n \tau+i n \sigma}, \quad \delta \varphi=\eta \mathrm{e}^{-n \tau+i n \sigma}
$$

into the equations of motion (3.2) and linearizing in $\xi$ and $\eta$, we get:

$$
\begin{aligned}
-\left(\sin ^{2} \theta \dot{\eta}\right)^{\cdot}+n \sin ^{2} \theta \dot{\eta}+n\left(\sin ^{2} \theta \eta\right)^{\cdot}+2 i \chi n \cot \theta \xi-2 i \chi(\cot \theta \xi)^{\cdot} & =0 \\
-\ddot{\xi}+2 n \dot{\xi}+\chi^{2}\left(1-\cot ^{4} \theta\right) \xi+2 i \chi \cot \theta \dot{\eta}-2 i \chi n \cot \theta \xi & =0,
\end{aligned}
$$

where $\cos \theta$ is the leading-order solution (A.2).

There are two possible regimes: (I) $\tau \rightarrow \infty$, finite $\chi$ and (II) $\tau \rightarrow \infty, \chi \rightarrow 0, \chi \tau$-fixed. In case (I) we can set $\theta=\pi / 2$, which yields

$$
\xi=\text { const } \mathrm{e}^{\left(n-\sqrt{n^{2}+\chi^{2}}\right) \tau}, \quad \eta=\text { const. }
$$

In case (II) there is a scaling region $\tau \sim 1 / \chi$, where $\xi=\xi(\chi \tau)$ and $\eta=\eta(\chi \tau)$. We can then neglect the terms of order $\chi^{2}$ (and keep those of order $\chi$ ) in the linearized equations. In particular we can neglect the second derivatives in $\tau$. The equations then are of the first order and can be integrated:

$$
\begin{aligned}
& \xi=\sin \theta\left(\frac{C_{1}}{1-\cos \theta}+\frac{C_{2}}{1+\cos \theta}\right) \\
& \eta=i\left(\frac{C_{1}}{1-\cos \theta}-\frac{C_{2}}{1+\cos \theta}\right)
\end{aligned}
$$


The constants of integration $C_{1}, C_{2}$ can be expressed in terms of $\partial \theta_{0}, \partial \varphi_{0}$ by setting $\chi=0$, when $\theta \rightarrow \theta_{0}$ and $\xi \rightarrow \partial \theta_{0}, \eta \rightarrow \partial \varphi_{0}$. Finally, we can write the solution of the linearized equations in the form that is valid both in the regime (I) and (II) by simply multiplying $\xi$ in (A.6) by $\mathrm{e}^{\left(n-\sqrt{n^{2}+\chi^{2}}\right) \tau}$, which is equivalent to 1 in the regime (II). This gives the solution (3.8) quoted in the main text.

Open Access. This article is distributed under the terms of the Creative Commons Attribution Noncommercial License which permits any noncommercial use, distribution, and reproduction in any medium, provided the original author(s) and source are credited.

\section{References}

[1] J.M. Maldacena, The large- $N$ limit of superconformal field theories and supergravity, Adv. Theor. Math. Phys. 2 (1998) 231 [Int. J. Theor. Phys. 38 (1999) 1113] [hep-th/9711200] [SPIRES].

[2] S.S. Gubser, I.R. Klebanov and A.M. Polyakov, Gauge theory correlators from non-critical string theory, Phys. Lett. B 428 (1998) 105 [hep-th/9802109] [SPIRES].

[3] E. Witten, Anti-de Sitter space and holography, Adv. Theor. Math. Phys. 2 (1998) 253 [hep-th/9802150] [SPIRES].

[4] A. Rej, Integrability and the AdS/CFT correspondence, J. Phys. A 42 (2009) 254002 [arXiv: 0907.3468] [SPIRES].

[5] D. Serban, Integrability and the AdS/CFT correspondence, arXiv:1003.4214 [SPIRES].

[6] V.G.M. Puletti, On string integrability. A journey through the two- dimensional hidden symmetries in the AdS/CFT dualities, Adv. High Energy Phys. 2010 (2010) 471238 [arXiv: 1006.3494] [SPIRES].

[7] D.Z. Freedman, S.D. Mathur, A. Matusis and L. Rastelli, Correlation functions in the CFT $(d) / A d S(d+1)$ correspondence, Nucl. Phys. B 546 (1999) 96 [hep-th/9804058] [SPIRES].

[8] G. Chalmers, H. Nastase, K. Schalm and R. Siebelink, R-current correlators in $N=4$ super Yang-Mills theory from anti-de Sitter supergravity, Nucl. Phys. B 540 (1999) 247 [hep-th/9805105] [SPIRES].

[9] S. Lee, S. Minwalla, M. Rangamani and N. Seiberg, Three-point functions of chiral operators in $D=4, N=4 S Y M$ at large- $N$, Adv. Theor. Math. Phys. 2 (1998) 697 [hep-th/9806074] [SPIRES].

[10] G. Arutyunov and S. Frolov, Some cubic couplings in type IIB supergravity on $A d S_{5} \times S^{5}$ and three-point functions in SYM(4) at large-N, Phys. Rev. D 61 (2000) 064009 [hep-th/9907085] [SPIRES].

[11] S. Lee, $A d S_{5} / C F T_{4}$ Four-point Functions of Chiral Primary Operators: Cubic Vertices, Nucl. Phys. B 563 (1999) 349 [hep-th/9907108] [SPIRES].

[12] S.S. Gubser, I.R. Klebanov and A.M. Polyakov, A semi-classical limit of the gauge/string correspondence, Nucl. Phys. B 636 (2002) 99 [hep-th/0204051] [SPIRES].

[13] S. Frolov and A.A. Tseytlin, Multi-spin string solutions in $A d S_{5} \times S^{5}$, Nucl. Phys. B 668 (2003) 77 [hep-th/0304255] [SPIRES]. 
[14] A.A. Tseytlin, Spinning strings and AdS/CFT duality, hep-th/0311139 [SPIRES].

[15] J. Plefka, Spinning strings and integrable spin chains in the AdS/CFT correspondence, Living Rev. Rel. 8 (2005) 9 [hep-th/0507136] [SPIRES].

[16] E.I. Buchbinder, Energy-Spin Trajectories in $A d S_{5} \times S^{5}$ from Semiclassical Vertex Operators, JHEP 04 (2010) 107 [arXiv:1002.1716] [SPIRES].

[17] R.A. Janik, P. Surowka and A. Wereszczynski, On correlation functions of operators dual to classical spinning string states, JHEP 05 (2010) 030 [arXiv:1002.4613] [SPIRES].

[18] E.I. Buchbinder and A.A. Tseytlin, On semiclassical approximation for correlators of closed string vertex operators in AdS/CFT, JHEP 08 (2010) 057 [arXiv: 1005.4516] [SPIRES].

[19] M.S. Costa, R. Monteiro, J.E. Santos and D. Zoakos, On three-point correlation functions in the gauge/gravity duality, arXiv:1008.1070 [SPIRES].

[20] D.E. Berenstein, R. Corrado, W. Fischler and J.M. Maldacena, The operator product expansion for Wilson loops and surfaces in the large- $N$ limit, Phys. Rev. D 59 (1999) 105023 [hep-th/9809188] [SPIRES].

[21] A. Polyakov, Old and new aspects of the gauge/strings correspondence, talk at Strings 2002, http://www.damtp.cam.ac.uk/strings02/avt/polyakov/.

[22] J. de Boer, H. Ooguri, H. Robins and J. Tannenhauser, String theory on AdS $S_{3}$, JHEP 12 (1998) 026 [hep-th/9812046] [SPIRES].

[23] A. Tsuji, Holography of Wilson loop correlator and spinning strings, Prog. Theor. Phys. 117 (2007) 557 [hep-th/0606030] [SPIRES].

[24] K. Peeters, J. Plefka and M. Zamaklar, Splitting strings and chains, Fortsch. Phys. 53 (2005) 640 [hep-th/0501165] [SPIRES].

[25] S. Dobashi and T. Yoneya, Resolving the holography in the plane-wave limit of AdS/CFT correspondence, Nucl. Phys. B 711 (2005) 3 [hep-th/0406225] [SPIRES].

[26] L.F. Alday, D. Gaiotto, J. Maldacena, A. Sever and P. Vieira, An Operator Product Expansion for Polygonal null Wilson Loops, arXiv:1006.2788 [SPIRES].

[27] H.J. Kim, L.J. Romans and P. van Nieuwenhuizen, The Mass Spectrum of Chiral N=2 D=10 Supergravity on $S^{5}$, Phys. Rev. D 32 (1985) 389.

[28] A.M. Polyakov, Gauge fields and space-time, Int. J. Mod. Phys. A 17S1 (2002) 119 [hep-th/0110196] [SPIRES].

[29] A.A. Tseytlin, On semiclassical approximation and spinning string vertex operators in $A d S_{5} \times S^{5}$, Nucl. Phys. B 664 (2003) 247 [hep-th/0304139] [SPIRES].

[30] G. Arutyunov and S. Frolov, Scalar quartic couplings in type IIB supergravity on $A d S_{5} \times S^{5}$, Nucl. Phys. B 579 (2000) 117 [hep-th/9912210] [SPIRES].

[31] E. D'Hoker, D.Z. Freedman and W. Skiba, Field theory tests for correlators in the AdS/CFT correspondence, Phys. Rev. D 59 (1999) 045008 [hep-th/9807098] [SPIRES].

[32] F. Gonzalez-Rey, B. Kulik and I.Y. Park, Non-renormalization of two point and three point correlators of $N=4 S Y M$ in $N=1$ superspace, Phys. Lett. B 455 (1999) 164 [hep-th/9903094] [SPIRES].

[33] K.A. Intriligator and W. Skiba, Bonus symmetry and the operator product expansion of $N=4$ super-Yang-Mills, Nucl. Phys. B 559 (1999) 165 [hep-th/9905020] [SPIRES]. 
[34] B. Eden, P.S. Howe and P.C. West, Nilpotent invariants in $N=4 S Y M$, Phys. Lett. B 463 (1999) 19 [hep-th/9905085] [SPIRES].

[35] A. Petkou and K. Skenderis, A non-renormalization theorem for conformal anomalies, Nucl. Phys. B 561 (1999) 100 [hep-th/9906030] [SPIRES].

[36] C. Kristjansen, J. Plefka, G.W. Semenoff and M. Staudacher, A new double-scaling limit of $N=4$ super Yang-Mills theory and PP-wave strings, Nucl. Phys. B 643 (2002) 3 [hep-th/0205033] [SPIRES].

[37] D.E. Berenstein, J.M. Maldacena and H.S. Nastase, Strings in flat space and pp waves from $N=4$ super Yang-Mills, JHEP 04 (2002) 013 [hep-th/0202021] [SPIRES].

[38] V.A. Kazakov, A. Marshakov, J.A. Minahan and K. Zarembo, Classical/quantum integrability in AdS/CFT, JHEP 05 (2004) 024 [hep-th/0402207] [SPIRES].

[39] N. Beisert, V.A. Kazakov and K. Sakai, Algebraic curve for the SO(6) sector of AdS/CFT, Commun. Math. Phys. 263 (2006) 611 [hep-th/0410253] [SPIRES].

[40] N. Dorey and B. Vicedo, On the dynamics of finite-gap solutions in classical string theory, JHEP 07 (2006) 014 [hep-th/0601194] [SPIRES].

[41] S. Frolov and A.A. Tseytlin, Rotating string solutions: AdS/CFT duality in non-supersymmetric sectors, Phys. Lett. B 570 (2003) 96 [hep-th/0306143] [SPIRES].

[42] N. Beisert, J.A. Minahan, M. Staudacher and K. Zarembo, Stringing spins and spinning strings, JHEP 09 (2003) 010 [hep-th/0306139] [SPIRES].

[43] K. Zarembo, Open string fluctuations in $A d S_{5} \times S^{5}$ and operators with large $R$ charge, Phys. Rev. D 66 (2002) 105021 [hep-th/0209095] [SPIRES].

[44] V. Pestun and K. Zarembo, Comparing strings in $A d S_{5} \times S^{5}$ to planar diagrams: An example, Phys. Rev. D 67 (2003) 086007 [hep-th/0212296] [SPIRES].

[45] A. Grossardt and J. Plefka, One-Loop Spectroscopy of Scalar Three-Point Functions in planar $N=4$ super Yang-Mills Theory, arXiv:1007.2356 [SPIRES]. 